\title{
REINHARDTIA ELEGANS MART. (ARECACEAE): UNA PALMA ENDÉMICA DEL BOSQUE MESÓFILO DE MONTAÑA MEXICANO
}

\author{
Guillermo lbarRa-Manríquez \\ Centro de Investigaciones en Ecosistemas \\ Universidad Nacional Autónoma de México \\ Antigua carretera a Pátzcuaro No. 8701 \\ Col. San José de la Huerta \\ 58190 Morelia, Michoacán, México \\ y \\ Ana Mendoza \\ Departamento de Ecología Evolutiva \\ Instituto de Ecología \\ Universidad Nacional Autónoma de México \\ Apartado Postal 70-275 \\ 04510 México, D.F.

\section{RESUMEN}

Se registra la presencia de Reinhardtia elegans Mart. (Arecaceae), una palma endémica de algunos bosques mesófilos de montaña de Oaxaca y Chiapas, cuya importancia no ha sido debidamente resaltada en los trabajos florísticos del sureste de México. Se aporta información sobre algunos aspectos del hábitat y estructura poblacional de la especie en la región de La Chinantla, Oaxaca, México. Reinhardtia.

Palabras clave: Arecaceae, bosque mesófilo de montaña, Chiapas, México, Oaxaca, palmas,

\section{ABSTRACT}

Reinhardtia elegans Mart. (Arecaceae) is a Mexican endemic species occurring in some cloud forests of Oaxaca and Chiapas, whose importance has been poorly documented in floristic studies of southeastern Mexico. We provide information about its habitat and population structure in La Chinantla region in Oaxaca, Mexico.

Key words: Arecaceae, cloud forest, Chiapas, Mexico, Oaxaca, palms, Reinhardtia. 


\section{INTRODUCCIÓN}

La familia Arecaceae ha sido considerada como un grupo de plantas bien definido, cuyos miembros son reconocidos con relativa facilidad. Entre las monocotiledóneas, la riqueza taxonómica del grupo es notable y sólo es menor a la documentada para las familias Liliaceae, Orchidaceae y Poaceae (Heywood, 1978). Las arecáceas están constituidas por 198 a 212 géneros y entre 2,650 a cerca de 3,000 especies, las cuales se distribuyen prácticamente en todas las áreas tropicales y subtropicales del Neotrópico y Paleotrópico, siendo su mayor centro de riqueza el sureste de Asia y con una menor representación de especies en África (Heywood, 1978; Cronquist, 1981; Uhl y Dransfield, 1987; Mabberley, 1993; Moraes et al., 1995). En México, la familia se encuentra representada por 22 géneros (Uhl y Dransfield, 1987), sobresaliendo en diversidad específica Chamaedorea, con alrededor de 45 especies (Hodel, 1992).

Las palmas son también una familia envuelta en una compleja problemática taxonómica que hace difícil la circunscripción de las subfamilias, géneros o especies (p. ej. Dahlgren et al., 1985; Uhl y Dransfield, 1987; Hodel, 1992; Henderson et al., 1995). Un elemento importante para justificar lo anterior es que este grupo tiene una deficiente representación en los herbarios, ya que en general se le omite en los trabajos de prospección florística. Lo anterior se debe principalmente a que la colecta de ejemplares de herbario es muy impráctica. Además, muchas de sus especies poseen individuos de gran tamaño, densidades poblacionales reducidas y/o una distribución geográfica muy restringida, lo que dificulta localizarlas y caracterizarlas adecuadamente (ver por ejemplo Hodel, 1992; Henderson et al., 1995).

El género Reinhardtia, constituido por seis especies ( $R$. elegans Liebm., $R$. gracilis (H. Wendl.) Burret, R. koschnyana (H. Wendl. \& Dammer) Burret, R. latisecta $(\mathrm{H}$. Wendl.) Burret, R. paiewonskiana Read, Zanoni \& Mejia y R. simplex $(\mathrm{H}$. Wendl.) Burret, es un buen ejemplo de la falta de representatividad de sus especies en los herbarios, si bien esta última condición es menos evidente para $R$. gracilis. La distribución del género es fundamentalmente mesoamericana, aunque de acuerdo con Henderson et al. (1995), dos especies se han encontrado en el noroeste de Colombia (Cuadro 1). Reinhardtia paiewonskiana es la única presente en el área antillana, a la cual se encuentra restringida (Read et al., 1987; Henderson, 2002). En México se han registrado tres especies que muestran una distribución hacia la vertiente atlántica del sureste del país: $R$. elegans (Chiapas y Oaxaca), $R$. gracilis (Chiapas, Oaxaca, Tabasco y Veracruz) y $R$. simplex (Chiapas), esta última citada recientemente para la región Lacandona, Chiapas, por Martínez et al. (1994). Tales plantas habitan básicamente en comunidades de bosque mesófilo de montaña y bosque tropical perennifolio, en sitios que no sobrepasan los 1,600 m de altitud (Moore, 1957a, b; Ibarra-Manríquez, 1988; Long y Heat, 1991; Martínez et al., 1994; Quero, 1994; Henderson et al., 1995; Henderson, 2002).

El presente trabajo forma parte de una investigación sobre la demografía comparada de las especies mexicanas de Reinhardtia. En particular, en este estudio se dan a conocer aspectos del hábitat y atributos demográficos de una población de $R$. elegans Mart., censada y colectada recientemente en la región de La Chinantla, Oaxaca y cuya presencia había pasado inadvertida en los trabajos florísticos realizados en la parte sureste de México. 
Ibarra-Manríquez y Mendoza: Reinhardtia elegans, una palma endémica del bosque mesófilo

Cuadro 1. Distribución geográfica de las especies de Reinhardtia. Abreviaturas de las especies: ELE (R. elegans Liebm.), GRA (R. gracilis (H. Wendl.) Burret), KOS (R. koschnyana (H. Wendl. \& Dammer) Burret, LAT ( $R$. latisecta (H. WEndl.) Burret), PAI (R. paiewonskiana Read, Zanoni \& Mejia), SIM (R. simplex (H. Wendl.) Burret).

\begin{tabular}{|c|c|c|c|c|c|c|}
\hline Subgéneros & \multicolumn{3}{|c|}{ Malortiea } & \multicolumn{3}{|c|}{ Reinhardtia } \\
\hline País & GRA & KOS & SIM & ELE & LAT & PAI \\
\hline México & . - & & . & . . & & \\
\hline Belice & . - & & & & . - & \\
\hline Guatemala & . - & & & & $?$ & \\
\hline Honduras & . . & . . &. & &. & \\
\hline Nicaragua & . & . & . . & & . - & \\
\hline Costa Rica & . & . . & . . & & . - & \\
\hline Panamá & &. & . . & & & \\
\hline Colombia & & . . &. & & & \\
\hline República Dominicana & & & & & & - \\
\hline
\end{tabular}

\section{IMPORTANCIA DEL REGISTRO DE R. ELEGANS EN MÉXICO}

La presencia de $R$. elegans en México fue indicada primeramente por Standley (1920-1926). Sin embargo, cabe destacar que a pesar del intenso trabajo florístico que se ha realizado en las últimas décadas por diversas instituciones botánicas en el sureste del país, $R$. elegans no ha sido colectada recientemente e incluso, no cuenta con ejemplares que documenten su presencia en los herbarios mexicanos. Esta especie no ha sido mencionada en los inventarios florísticos de Chiapas (p. ej. Breedlove, 1986, Long y Heat, 1991; Martínez et al., 1994), ni en las publicaciones similares producidas para Tabasco y Veracruz (Cowan, 1983; Sosa y Gómez-Pompa, 1994), estados que cuentan con localidades potenciales para su establecimiento. En su estudio monográfico de las palmas de Veracruz, Quero (1994) señala que esta especie probablemente se encuentra en dicho estado, citando como ejemplares de referencia material depositado en el herbario $\mathrm{BH}$ y una colecta supuestamente también de Oaxaca, perteneciente a I. Calzada (16410), la cual no se ha hallado en el herbario MEXU. Los esfuerzos realizados por diversas instituciones botánicas en el área mesoamericana tampoco han encontrado localidades adicionales para $R$. elegans. El registro de esta especie indicado por Henderson et al. (1995) y Henderson (2002) para el distrito de Olancho, Honduras, es de dudosa validez, ya que el ejemplar que aparentemente lo respalda no ha sido localizado en el herbario K (A. Henderson, com. pers.). 
En 1996 se localizó una población de la especie en los alrededores del poblado de Santo Domingo Lataní, municipio de Santiago Choapam, lo que resulta un hallazgo interesante, ya que de acuerdo con Moore (1957a), la última colecta de esta palma había sido realizada en Oaxaca, en 1946, por E. Hernández-X. (número de colecta 1245) y J. Sharp. El mencionado autor también indica que la especie es endémica de México, conocida de Oaxaca (municipios de Ixtlán de Juárez, Niltepec, Santiago Choapam) y de Chiapas, citando una localidad de ubicación incierta ("mountains near Fenix"). Esta área, conocida actualmente como Rancho Fénix, está situada al este de la Hacienda Monserrate $\left(16^{\circ} 33^{\prime} \mathrm{N}, 94^{\circ} 00^{\prime} \mathrm{W}\right)$.

La población localizada recientemente se encuentra ubicada alrededor de $95^{\circ} 54^{\prime} 40^{\prime \prime} \mathrm{W}$ y $17^{\circ} 24^{\prime} 00^{\prime \prime} \mathrm{N}$, donde se desarrolla en altitudes entre 900-1,150 m s.n.m. Los ejemplares que certifican este registro (Guillermo Ibarra Manríquez 4045, 4046, 4070, $4071,4160,4161)$ serán depositados en los herbarios IEB, MEXU, MO y XAL. Hasta el momento, $R$. elegans ha sido encontrada exclusivamente como componente del bosque mesófilo de montaña, aunque no está incluida en el listado de géneros que poseen especies típicamente adscritas a este tipo de vegetación en México (Rzedowski, 1996).

El bosque mesófilo de montaña en la región de La Chinantla es una comunidad bastante heterogénea en sus atributos estructurales y en su composición florística (Meave et al., 1996). Reinhardtia elegans se establece en áreas muy inaccesibles, sobre suelos profundos, oscuros, aparentemente ricos en materia orgánica, sobre pendientes de hasta $40^{\circ}$. Otras especies presentes en este bosque son Abuta panamensis (Menispermaceae), Alfaroa mexicana (Juglandaceae), Brunellia mexicana (Brunelliaceae), Chamaedorea elegans (Arecaceae), Garcinia intermedia (Clusiaceae), Guarea glabra (Meliaceae), Liquidambar styraciflua (Hammamelidaceae), Matayba oppositifolia (Sapindaceae), Pseudolmedia spuria (Moraceae), Psychotria elata (Rubiaceae), Pouteria durlandii (Sapotaceae) y Ticodendron incognitum (Ticodendraceae). En los claros de la comunidad es posible encontrar árboles pioneros, de rápido crecimiento como Cecropia peltata (Cecropiaceae), así como Heliocarpus appendiculatus y Trichospermum mexicanum (Tiliaceae).

\section{ASPECTOS DEMOGRÁFICOS DE R. ELEGANS EN OAXACA}

Los individuos observados en la localidad de estudio poseen hábito cespitoso (con reproducción clonal) y sólo de manera muy infrecuente presentan un sólo "tronco", forma de crecimiento descrita por Moore (1957a, b). Esta palma aumenta de tamaño por la adición de nuevos tallos, mientras que cada uno de éstos incrementa su altura al producirse nuevas hojas. El tallo dominante alcanza hasta $5 \mathrm{~m}$ de altura, rodeado por 2-8(-18) "troncos" más pequeños, de hasta $1 \mathrm{~m}$. Ocasionalmente se encuentran palmas con 2 a 4 tallos relativamente altos, que se desarrollan cuando muere el eje principal, aunque eventualmente uno de ellos crece más y se convierte en el dominante.

Con el objeto de evaluar las condiciones demográficas actuales de la población de $R$. elegans, se delimitó un sitio de $20 \times 50 \mathrm{~m}$, en donde se marcaron a todos los individuos presentes en el área. A cada individuo se le registró el número total de tallos y la altura del tallo dominante. La población se clasificó en categorías de tamaño, las cuales se pueden describir más fácilmente por el número de tallos. 


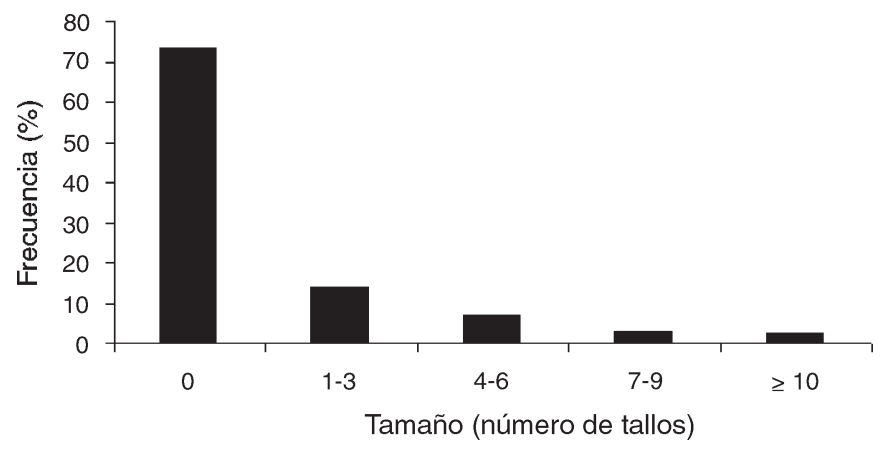

Fig. 1. Distribución de frecuencias del tamaño de los individuos (número de tallos) de Reinhardtia elegans en Santo domingo Lataní, Oaxaca.

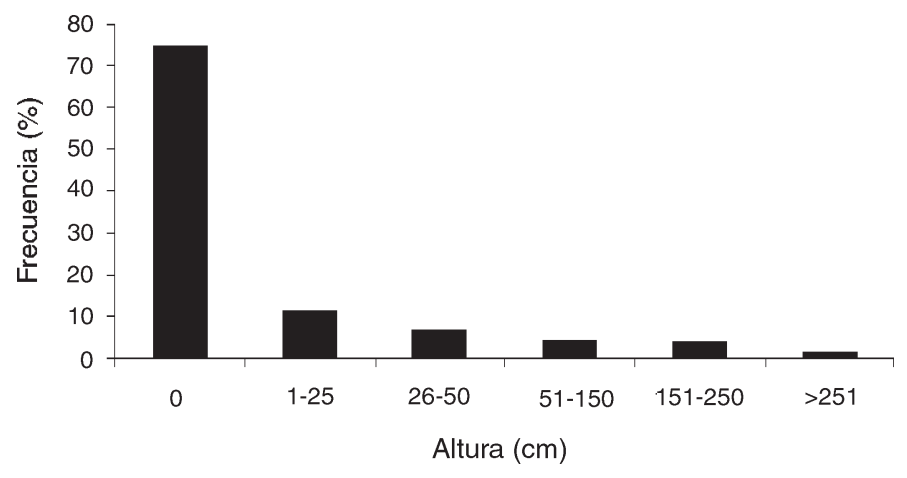

Fig. 2. Distribución de frecuencias de la altura del tallo más viejo de los individuos de Reinhardtia elegans en Santo Domingo Lataní, Oaxaca.

La estructura correspondiente muestra que la población se está regenerando de manera natural, como lo indica la forma de "J" invertida (Fig. 1), donde la mayoría de los individuos (74\%) son plántulas que aún no poseen un tallo definido. De la población, 14\% tiene 1-3 tallos y menos de $2 \%$ tiene más de 10. Las plántulas de origen sexual y los rebrotes, producto del crecimiento clonal, no tienen altura determinada y junto con éstos, los tallos de menor altura son los más abundantes en la población (Fig. 2). Las semillas 
de esta palma germinan y se establecen debajo o relativamente cerca de sus progenitores, ya que pueden detenerse entre la abundante hojarasca que se encuentra en el suelo, aun en aquellas poblaciones localizadas sobre pendientes pronunciadas.

Con base en los criterios propuestos por Rabinowitz (1981) y la información disponible hasta el momento, $R$. elegans es una palma rara, ya que presenta una distribución geográfica muy restringida, una estrecha amplitud ecológica y posiblemente un reducido número de poblaciones, situación que comparte con $R$. paiewonskiana (Read et al., 1987). A pesar de que no hemos generado información que documente de manera precisa la distribución y abundancia de $R$. elegans en el área de Santo Domingo Lataní, los datos proporcionados por los habitantes locales indica que esta especie cuenta con escasas poblaciones, muy distantes entre sí. De acuerdo con nuestras observaciones de campo, dentro de una población particular, existe un alto número de individuos en el sotobosque, siendo entonces la especie dominante en ese estrato.

En general puede apreciarse que $R$. elegans es una especie sensible al drástico cambio en el uso de suelo que está ocurriendo en La Chinantla, que ha provocado una deforestación intensa alrededor de los asentamientos humanos presentes en el área. El hombre está modificando el hábitat de esta palma a través del proceso de "roza, tumba y quema", con el propósito de ampliar las zonas de cultivo. La creciente intensidad de tal arrasamiento no permite que se den las condiciones y el tiempo necesario para que tal fenómeno de alteración del hábitat se revierta. Por lo anterior, es posible que ocurran extinciones locales que pueden llevar en el futuro a la desaparición de la especie en toda la región. El escenario para la conservación de este taxon es complejo, pues para los pobladores de la comarca no posee ninguna utilidad inmediata, a pesar de su enorme potencial como planta ornamental. Sin embargo, algunos habitantes indican que personas no residentes en el área han empezado a sustraer semillas de $R$. elegans con propósitos de uso hasta el momento desconocidos.

La recomendación más inmediata respecto a esta situación consiste en la conveniencia de realizar trabajos de investigación más detallados que permitan determinar, con mayor seguridad, el grado de amenaza que se cierne sobre la palma y establecer medidas que aseguren su conservación a largo plazo.

La presencia de esta especie endémica de México en hábitats muy particulares de La Chinantla, junto con otros elementos biogeográficamente interesantes, como por ejemplo, Cyrilla racemiflora, Cyrillaceae (Gallardo et al., 1998), Oreomunnea mexicana, Juglandaceae (Rzedowski y Palacios-Chávez, 1977) y Ticodendron incognitum, Ticodendraceae (Meave et al.,1996), sin duda contribuirá a entender la compleja historia de los bosques mesófilos de México.

\section{AGRADECIMIENTOS}

Deseamos agradecer a los residentes del pueblo de Santo Domingo Lataní, Oaxaca, por habernos permitido trabajar en sus interesantes bosques, aunque especialmente a Alejandro García Gómez, ya que fue la primera persona de la comunidad dispuesta a apoyarnos en cuestiones logísticas y de trabajo de campo. Nuestro reconocimiento a Daniel Ocaña Nava, Aldo Valera Vázquez, Luis Alamán, Carlos Ramos Guerrero y Pedro Zaragoza García por su importante ayuda en el difícil trabajo de campo. 
Ibarra-Manríquez y Mendoza: Reinhardtia elegans, una palma endémica del bosque mesófilo

La asistencia de María de Lourdes Rico, Andrew Henderson, Cecilia Sánchez Garduño, Francisco G. Lorea Hernández y Oswaldo Téllez Valdez, fue muy valiosa para obtener diversa información sobre Reinhardtia de los herbarios BM, K, MEXU y XAL. Este estudio fue financiado por el Consejo Nacional de Ciencia y Tecnología (CONACyT), con número $3721 \mathrm{P}-\mathrm{N}$. Finalmente, agradecemos los valiosos comentarios aportados al trabajo por dos revisores anónimos, los cuales mejoraron el contenido del manuscrito.

\section{LITERATURA CITADA}

Breedlove, D. E.1986. Listados florísticos de México IV. Flora de Chiapas. Instituto de Biología, Universidad Nacional Autónoma de México. México, D. F. 246 pp.

Cowan, C. P. 1983. Listados florísticos de México I. Flora de Tabasco. Instituto de Biología, Universidad Nacional Autónoma de México. México, D. F. 123 pp.

Cronquist, A. 1981. An integrated system of classification of flowering plants. Columbia University Press. Nueva York. 1262 pp.

Dahlgren, R. M. T., H. T. Clifford y P. F. Yeo. 1985. The families of the Monocotyledons. Structure, evolution, and taxonomy. Springer-Verlag. Berlin Heidelberg. 520 pp.

Gallardo, C., J. Meave y A. Rincón. 1998. Plantas leñosas raras del bosque mesófilo de montaña IV. Cyrilla racemiflora L. (Cyrillaceae). Bol. Soc. Bot. México 62: 183-186.

Henderson, A., G. Galeano y R. Bernal. 1995. Field guide to the palms of the Americas. Princeton University Press. Princeton. New Jersey. $352 \mathrm{pp}$.

Henderson, A. 2002. Phenetic and phylogenetic analysis of Reinhardtia (Palmae). Am. J. Bot. 89(9): 1491-1502.

Heywood, V. H. 1978. Flowering plants of the world. Oxford University Press. Oxford. 335 pp.

Hodel, R. H. 1992. Chamaedorea palms. The species and their cultivation. Allen Press. Lawrence, Kansas. $338 \mathrm{pp}$.

Ibarra-Manríquez, G. 1988. The palms of a tropical rain forest in Veracruz, México. Principes 32(4): 147-155.

Long, A. y M. Heat. 1991. Flora of the El Triunfo Biosphere Reserve, Chiapas, México: a preliminary floristic inventory and the plant communities of Polygon I. Anales Inst. Biol. Univ. Nac. Autón. México, Ser. Bot. 62: 133-172.

Mabberley, D. J. 1993. The plant-book. A portable dictionary of the higher plants. Cambridge University Press. Cambridge. 707 pp.

Martínez, E., C. H. Ramos y F. Chiang. 1994. Lista florística de la Lacandona, Chiapas. Bol. Soc. Bot. México 54: 99-177.

Meave, J., C. Gallardo y A. Rincón. 1996. Plantas leñosas raras del bosque mesófilo de montaña II. Ticodendron incognitum Gómez-Laurito \& Gómez P. (Ticodendraceae). Bol. Soc. Bot. México 59: $149-152$.

Moraes, M., G. Galeano, R. Bernal, H. Balslev y A. Henderson. 1995. Tropical Andean palms (Arecaceae). In: Churchill, S. P., H. Balslev, E. Forero \& J. L. Luteyn (eds.). Biodiversity and conservation of neotropical montane forests. The New York Botanical Garden. Nueva York. pp. 473-487.

Moore Jr., H. E. 1957a. Reinhardtia. Gentes Herb. 8(7): 541-576.

Moore Jr., H. E. 1957b. The genus Reinhardtia. Principes 4(1): 127-145.

Quero, H. J. 1994. Flora de Veracruz. Fasciculo 81. Palmae. Instituto de Ecología A. C. \& University of California, Riverside. Xalapa. 118 pp.

Rabinowitz, D. H. 1981. Seven forms of rarity. In: Synge, H. (ed.). The biological aspects of rare plant conservation. J. Wiley \& Sons Ltd., Chichester. pp. 205-217. 
Read, R. W., T. A. Zanoni y M. Mejia. 1987. Reinhardtia paiewonskiana (Palmae), a new species for the West Indies. Brittonia 39(1): 20-25.

Rzedowski, J. 1996. Análisis preliminar de la flora vascular de los bosques mesófilos de montaña de México. Acta Bot. Mex. 35: 25-44.

Rzedowski, J. y R. Palacios-Chávez. 1977. El bosque de Engelhardtia (Oreomunnea) mexicana en la región de La Chinantla (Oaxaca, México): una reliquia del Cenozoico. Bol. Soc. Bot. México 36: 93-127.

Sosa, V. y A. Gómez-Pompa (comps.). 1994. Flora de Veracruz. Fascículo 82: Lista florística. Instituto de Ecología A. C. y University of California, Riverside. Xalapa. 245 pp.

Standley, P. C. 1920-26. Trees and shrubs of Mexico. Contr. U. S. Natl. Herb. 23: 1-1721.

Uhl, N. W. y J. Dransfield. 1987. Genera palmarum. A classification of palms based on the work of Harold E. Moore, Jr. Allen Press. Lawrence, Kansas. 610 pp.

Recibido en febrero de 2002.

Aceptado en enero de 2003. 